\title{
Empirical Determination of Total Atmospheric Refraction at Centimeter Wavelengths by Radiometric Means
}

\author{
Alan C. Anway \\ Contribution from Collins Radio Company, Cedar Rapids, Iowa
}

(Received October 5, 1962)

\begin{abstract}
The experimental procedures and data analysis methods used in the determination of total atmospheric refraction by radiometric means are described. The results of five months observation are presented in plots of the mean refraction, its standard deviation, and standard error of estimate for specified altitude angles between 2 and 65 degrees. The a.m. values of refraction are significantly greater than those of the p.m. at the same altitude angle. This effect is attributed to the diurnal cycle. The measured total refraction exhibits a strong linear correlation with surface refractivity
\end{abstract}

\section{Introduction}

Development of a precise radiometric sextant has made possible the measurement of the effect of the earth's atmosphere on the direction of propagation of $2-\mathrm{cm}$ wavelength radio waves. In particular, this paper considers the problem from a phenomenological point of view, and reports the performance and analysis of empirical observations over a wide range of observed altitude angles and for the variety of weather conditions found in nature. Refractive bending effects in the vertical plane (that is, the altitude coordinate) are considered since, under the usual assumption of spherical stratification, azimuthal refraction does not exist. Further, ionospheric effects are assumed negligible at $2-\mathrm{cm}$ wavelengths; therefore, only tropospheric phenomena are being considered.

Smart [1956] has shown that, in general, atmospheric refraction depends on the index of refraction profile. However, if the observed altitude is restricted to relatively high angles (that is, greater than $20 \mathrm{deg}$ ), total atmospheric refraction is found to depend only on the surface value of refractivity and the observed altitude angle. Extension of the analytical consideration to low-altitude angles becomes very complex, and generally ray-tracing techniques have been followed in this case. The usual procedure is to measure the refractivity profile with a radiosonde instrument and then compute, from this profile, the total atmospheric refraction by numerical integration methods [Shulkin, 1952].

In spite of the wide use of computed values of atmospheric refraction, it appears that little information is available on precise measurements of this quantity at radio wavelengths. Anderson et al. [1960] used a beacon tracking radar to measure lowangle refraction through part of the atmosphere. Experimental studies of very low-angle (horizontal) refraction through the entire atmosphere have been performed by Aarons et al. [1958].

It is believed that the investigation reported herein represents the first attempt to precisely meas- ure total atmospheric microwave refraction over a wide range of altitude angles and with the normal variation of meteorological parameters of the atmosphere. To the knowledge of the author, the only studies which closely approach the present one were those of Marner and Ringcen [1956], and Marner and Stewart [1955] at $8.7 \mathrm{~mm}$. Ringoen et al., [1952] did perform some measurements at $1.91 \mathrm{~cm}$ with a radio sextant of significantly lower precision than the one used in this study. However, the refinements of equipment and analysis technique used here are believed important enough to make this study unique.

The plan was to use a radiometric sextant to track the sun as it traversed the sky, and to record the observed values of the sun's altitude angle at certain precisely determined times throughout the day. The measurements were obtained at Cedar Rapids, Iowa, between August and December 1959. The observed altitude angles were limited by ground effects and by the maximum observed solar altitude angle to within 2 and $65 \mathrm{deg}$ respectively. The U.S. Naval Observatory made available highly accurate ephemeris data from which the sun's position was computed for any specific time for which a radio sextant observation was made. These computed values were conceived to be true values, and any deviation from them was due to error-producing causes in the equipment, the atmosphere, or the center of microwave radiation from the celestial source. The difference between the observed and true altitude angle was the total error produced by all causes, among them atmospheric refraction. Fortunately, atmospheric refraction was the largest of all errors, so its effect was distinguished easily.

Operation of a radio sextant system depends on microwave radiation emanating from the sun. In the $2-\mathrm{cm}$ microwave region, the sun radiates thermal noise energy similar to a blackbody of temperature approximately $7,000^{\circ} \mathrm{K}$. The space surrounding the sun also radiates a very minute amount of energy and appears as a blackbody of temperature very near absolute zero. 
A sensitive microwave receiver connected to a highly directional antenna senses when the sun is in the antenna beam by an increase in noise output from the receiver. If the sun were directly centered in the beam, the noise output would be maximum, and if the sun were displaced slightly from boresight, the output would be reduced. Assuming symmetry of the antenna beam and of the temperature distribution of the source, circular scanning of the sun's disk with the antenna beam can provide tracking error information. That is, if the sun is centered in the scan pattern, no change in d-c output level is obtained over the scan cycle, since the beam always sees the same effective source temperature. If the sun is not centered on the scan axis, the d-c output is modulated at the scan rate and correlated (phased) with the direction of the scan-axis to source-center displacement. The modulation arises since during part of the scan cycle the beam predominantly sees the cold space, and during the remaining part of the cycle it sees the hot sun.

After suitable a-c amplification, the modulated signal is synchronously detected to provide a pair of $\mathrm{d}$-c voltages, one in up-down, the other in right-left coordinates, which are proportional in magnitude and polarity to the magnitude and direction of the sun's displacement in line-of-sight coordinates. These signals are used to achieve automatic tracking.

An air-supported radome was used to provide a temperature controlled environment for the sextant and to protect it from the effects of weather and wind. This radome was made by cementing sections of neoprene-coated nylon fabric into the shape of a truncated sphere. It was fastened at its base to a circular ring which was free to rotate in the horizontal plane. The ring was driven by an electric motor so that the radome rotated $360 \mathrm{deg}$ in azimuth every $20 \mathrm{sec}$. Radome rotation was necessary to remove refractive errors produced by localized differences in the radome material. Rotating the radome caused rapid fluctuations in apparent position of the source which were effectively filtered, or averaged by the tracking-servo time constant. Thus, the sextant followed the mean apparent position of the source. There is adequate evidence beyond the scope of this paper that radome rotation successfully removed the effects of radome structural variations for altitude angles greater than $15 \mathrm{deg}$. Below this angle the effects are somewhat uncertain, and this analysis will consider them.

\section{Data Acquisition and Reduction}

We have the following mathematical model:

$$
h=h_{0}+\sum_{i=1}^{n} F_{h i}(x, y, \ldots)+R_{h}(a, b, \ldots),
$$

where $h$ is the true altitude angle, and $h_{0}$ is the observed altitude angle. $F_{h i}(x, y, \ldots)$ are functional relations which describe predictable (systematic and assignable) errors in the altitude coordinate, that is, those errors that are assignable to known causes, and are known functions of the several independent variables $x, y, \ldots$ and perhaps the dependent variable $h . R_{h}(a, b, \ldots)$ is the residual error in the altitude coordinate, and is some unknown function of the infinite variety of independent variables $a, b, \ldots$ That is, $R_{h}(a, b, \ldots)$ is random in that we cannot predict its precise value from a knowledge of only a finite number of independent variables.

To arrive at a model which is useful for the digital computations performed in this analysis (1) must be expressed in discrete, rather than analog form. Thus, for the $j$ th observation, we have

$$
h_{j}=h_{0 j}+\Delta h_{1 j}+\Delta h_{2 j}+\ldots \Delta h_{k j}+e_{j},
$$

where $\Delta h_{k j}$ is the error due to the $k$ th assignable cause under the specific conditions existing at the instant the $j$ th observation was made. The residual random error at the instant of observation is $e_{j}$. In effect then, $\Delta h_{k j}$ is a correction term due to the $k$ th cause which must be added to the observed value of altitude angle to make it more nearly correct. For example, $\Delta h_{1 j}$ might be the correction due to atmospheric refraction and $\Delta h_{2 j}$ might be the correction due to a systematic error in the angular readout mechanism. For this analysis, the residual error is assumed to be normally distributed with mean value $(\mu)$ of zero and standard deviation, $\sigma$.

Let the first correction term, $\Delta h_{1}$, be the total measured atmospheric refraction, $\tau$. Then, from the true and observed altitude angles and the remaining correction terms, the atmospheric refraction can be computed from

$$
\tau_{i}+e_{j}=h_{j}-h_{0 j}-\Delta h_{2 j}-\ldots \Delta h_{k j} .
$$

As long as the conditions assumed for $e_{j}$ hold, (3) is an unbiased estimate of the true value of measured refraction. Notice that refraction cannot be determined perfectly, it can only be estimated to within a standard error of $\sigma$.

The problem in making precise determinations of atmospheric refraction by this method reducesto one of evaluating the various errors introduced by the measuring instrument and by the sun.

An angular error can be introduced into the measurement of solar altitude angle if the distribution of intensity of microwave radiation from the sun should become asymmetric with respect to its center. Such asymmetry can result from enhanced radiation in localized regions of flare or sunspot activity. The time and location of the occurrence of an active region on the sun's disk was not predictable, but the fact of its occurrence was detectable with the radio sextant and by other independent observations. Thus, intervals when dynamic solar activity effects were present could be identified and were rejected from the sample of observations used for the determination of refraction. Fortunately, the frequency and duration of solar activity at the observational wavelength were small and relatively little data were lost. 
Ideally, it would be desirable to determine the instrument error corrections by independent techniques. Unfortunately no such determination of the readout dial-index error could be implemented which would have the desired accuracy. Therefore, it was necessary to evaluate this error by first eliminating all other errors from the data. The dial-index error is independent of altitude angle so evaluation of the remaining errors was required only over a small range of angles or, at the least, some specific altitude angle.

Fortunately, total atmospheric refraction can be accurately determined for high-altitude angles by computation requiring only surface measured information. This permitted removal of the largest of the error components and revealed the remaining small instrument errors which were determinable by independent means. After the various error components were evaluated, the process was reversed and (3) was used to compute the total refraction for all angles of observation.

It was found that there were three significant sources of tracking errors within the instrument itself. They are listed and discussed below.

(1) Outer-loop bias error - a nonconstant, uncontrolled, assignable error arising from residual biases in the outer-loop of the tracking-servo. This error was considered to be constant over various predetermined time intervals and varied from interval to interval. Its magnitude was determined from the synchronous detector output recordings. That is, the output voltage recordings were examined to determine both the time interval over which the output voltage was considered to have a given average value, and the value of that average. The angular offset was calculated by dividing this value by the voltage-angle system analog. This analog was determined periodically throughout the entire period of tracking activity. The angular outer-loop offset errors, so determined, were used to correct the sextant's indicated altitude angle.

(2) Readout transmission error - a sinusoidal function of observed altitude angle which arises from slight differences in the gain and phase shift of the 10-kc transmission line between the altitude axis angle sensing synchro and its follow-up resolver. This error was known to be of the form $A$ sin $\left(360 h_{0}+\phi\right)$. Over the time interval between adjustments of the gain and phase controls it was specified by determining the constants $A$ and $\phi$. The phaseangle constant was estimated by observation of graphical plots. A linear correlation of the residual tracking errors and the mathematical function $\sin \left(360 h_{0}+\phi\right)$ was performed. Several trials were made in which different estimated values of the phase angle were used. The phase angle yielding the largest correlation coefficient was considered the proper one to specify the readout transmission error. The amplitude constant, $A$, was given by the slope of the linear regression line. The value of this error was computed for every tracking observation and the indicated altitude was corrected by this value.
(3) Dial index error - a constant value arising from error in setting the readout dial with respect to the boresight axis. This error is considered to be fixed throughout the intervals between adjustment of the dials and/or mechanical failure or adjustment which would change the relationship between the dial and the boresight axis. This error was determined by computing the average tracking error (corrected for calculated high-angle refraction and outer-loop error) throughout the entire interval, the averaging being done for all observed altitude angles greater than $20 \mathrm{deg}$. Again, each tracking observation was corrected by the appropriate dial index value.

Throughout the tracking period there were certain contaminating influences which further affected the measurements. These contaminations were of such a nature that they did not readily permit determination of the amount of error and therefore the data could not be corrected with any degree of accuracy. Observations known to have been made under the influence of these uncorrectable effects were rejected from further consideration in the determination of atmospheric refraction. The following conditions specify the criteria for rejection of data.

(1) Occurrence of dynamic solar activity.

(2) Radome not rotating.

(3) Vertical axis maladjustment.

(4) Periods of known abnormally large wind and temperature effects resulting from removal or addition of the radome.

(5) Improper operation of certain components. The rejected interval was usually prior to complete failure or necessary readjustment of the component.

(6) Transient tracking conditions; during acquisition, reacquisition (after momentary power interruption), radiometer operation, or tracking through an obstruction to the line-of-sight.

(7) Outer-loop offset-correction information inadequate to compensate properly for this error source.

(8) Data known to be bad at the time of observation, and so labeled prior to computation of tracking error.

The above data rejections eliminated observations which deviated significantly from the normal mode of operation, and thus tended to bring the observations into statistical control.

There were approximately 40,000 observations made over a five-month interval which were selected and corrected for the three instrument errors and which were suitable for the analysis of atomospheric refraction.

The observations of apparent altitude angle were made at $1-\mathrm{min}$ intervals and hence were, in general, for nonintegral values of the observed angle. For this study, refraction was to be determined for the following observed altitude angles: $2,4,6,8,10,14$, $18,22,26,30,35,40,45,55,60$, and 65 deg. Thus, it was necessary to select the desired values of measured refraction from the entire collection of corrected observations. This was done by scrutinizing the the data and listing all those observations whose observed altitude angle was within $\pm 0.09 \mathrm{deg}$ of the specified integral values. In cases where there 
were more than one observation in this specified small interval, the one nearest the desired altitude was used. Surface refractivity, time of day, and status of the radome were listed along with measured refraction.

Before proceeding with the averaging process at each desired altitude angle, it was necessary to determine whether or not there were differences in refraction at the same observed angle in the morning (a.m.) and the afternoon (p.m.). The data for $30 \mathrm{deg}$ were examined, and all the a.m.-p.m. pairs with the radome on, were selected. Ten pairs were obtained and the difference between the a.m. sample average and the p.m. sample average was computed. This difference was tested for significance at the 5-percent level with the well-known $t$-test for significance of means when the variances are known to be unequal. The test showed no significance, so it was repeated at $8 \mathrm{deg}$ and $18 \mathrm{deg}$. The same result was obtained. It was felt that an inordinately large variance contributed to the lack of significance. Further examination revealed that the pairs of samples obtained covered a time span of several months and hence there were large variations in surface refractivity. The test was repeated, using pairs taken only from the month of October. Angles of 10, 14, and $35 \mathrm{deg}$ were added. The tests were now all significant except those at 8 deg which had only 4 pairs of observations and at $14 \mathrm{deg}$ which was very nearly significant. Thus, it was concluded that there was a real difference between the a.m. and p.m. values of refraction, and therefore, the averages must be obtained for both sets of altitude angles.

In addition to the effect of a.m. and p.m., there was a question of whether or not the radome introduced a refractive effect of its own. Two different tests were employed to answer this question. The first was to simply test for a significant difference between a sample of observations with radome on and another sample of observations with radome off, when both samples were obtained for the p.m. altitude angle of $6 \mathrm{deg}$. In this case, the conclusion was that there is no significant difference in refraction with the radome on over that with the radome off. A second test considered a pair of observations (that is, radome on and off) as being the results of two measurements on the same test specimen when the specimen had been subjected to two different treatments; namely, radome on and radome off. It was attempted to make the individual specimens as homogeneous as possible by using p.m. conditions which had nearly ( $\pm 1 \quad N$-unit) the same surface refractivity. The differences between the refraction with radome off and radome on were determined for 10 different specimens and the average difference was tested for statistical significance from zero. The result was that the average difference did not signifcantly differ from zero and therefore it was concluded that the radome did not introduce an important refractive effect of its own.

These two preliminary tests provided the information necessary to decide how the observed refractions should be grouped in order to obtain the best estimate of the average atmospheric refraction during the test period. That is, the observations must be separated into two groups depending on whether the desired altitude angle was obtained in the a.m. or in the p.m. It was not necessary to further separate the observations into radome on and radome off groups, hence they were lumped together.

The final step in the computational process was to compute the mean, $\bar{\tau}$, standard error of the mean, $\sigma_{\bar{\tau}}$, and standard deviation, $\sigma_{\tau}$, of atmospheric refraction for each altitude angle, $h_{0}$.

\section{Discussion of Data and Results}

The results of computation of the statistics of refraction for each specified altitude angle are shown in table 1.

TABLE 1. Statistics of measured atmospheric refraction for specified altitude angles at $1.85 \mathrm{~cm}$

\begin{tabular}{|c|c|c|c|c|c|}
\hline$h_{\mathrm{o}}$ & $\bar{\tau}$ & $\boldsymbol{\sigma}_{\bar{\tau}}$ & $\sigma_{\tau}$ & $\begin{array}{l}\text { Number of } \\
\text { observations }\end{array}$ & $\alpha$ \\
\hline $\begin{array}{l}\quad \text { deg } \\
6^{\circ} \text { a.m } \\
8^{\circ} \\
10^{\circ} \\
14^{\circ} \\
18^{\circ} \\
22^{\circ} \\
26^{\circ} \\
30^{\circ} \\
35^{\circ} \\
40^{\circ} \\
45^{\circ} \\
55^{\circ} \\
60^{\circ} \\
65^{\circ} \mathrm{M} \mathrm{MP} \\
60^{\circ} \mathrm{p} \mathrm{pm} \\
55^{\circ} \\
45^{\circ} \\
40^{\circ} \\
35^{\circ} \\
30^{\circ} \\
26^{\circ} \\
22^{\circ} \\
18^{\circ} \\
14^{\circ} \\
10^{\circ} \\
8^{\circ} \\
6^{\circ} \\
4^{\circ} \\
2^{\circ}\end{array}$ & $\begin{array}{l}\text { deg } \\
-0.1702 \\
-.1344 \\
-.1069 \\
-.0759 \\
-.0608 \\
-.0480 \\
-.0403 \\
-.0348 \\
-.0301 \\
-.0274 \\
-.0230 \\
-.0171 \\
-.0152 \\
-.0112 \\
-.0129 \\
-.0153 \\
-.0205 \\
-.0237 \\
-.0262 \\
-.0328 \\
-.0384 \\
-.0465 \\
-.0565 \\
-.0745 \\
-.1052 \\
-.1300 \\
-.1689 \\
-.2342 \\
-.3658\end{array}$ & $\begin{array}{l}\text { deg } \\
\pm 0.0048 \\
\pm .0042 \\
\pm .0027 \\
\pm .0016 \\
\pm .0013 \\
\pm .0011 \\
\pm .0008 \\
\pm .0008 \\
\pm .0007 \\
\pm .0009 \\
\pm .0012 \\
\pm .0007 \\
\pm .0009 \\
\pm .0009 \\
\pm .0007 \\
\pm .0007 \\
\pm .0007 \\
\pm .0006 \\
\pm .0006 \\
\pm .0007 \\
\pm .0008 \\
\pm .0010 \\
\pm .0011 \\
\pm .0014 \\
\pm .0019 \\
\pm .0021 \\
\pm .0030 \\
\pm .0045 \\
\pm .0073\end{array}$ & \begin{aligned} \multicolumn{1}{l}{$d e g$} \\
11. $65 \times 10^{-3} \\
$ 13. $20 \times 10^{-3} \\
9.11 \times 10^{-3} \\
6.48 \times 10^{-3} \\
6.18 \times 10^{-3} \\
5.39 \times 10^{-3} \\
4.02 \times 10^{-3} \\
3.72 \times 10^{-3} \\
3.66 \times 10^{-3} \\
4.25 \times 10^{-3} \\
5.01 \times 10^{-3} \\
2.66 \times 10^{-3} \\
2.95 \times 10^{-3} \\
1.90 \times 10^{-3} \\
2.39 \times 10^{-3} \\
2.70 \times 10^{-3} \\
2.97 \times 10^{-3} \\
2.92 \times 10^{-3} \\
3.56 \times 10^{-3} \\
4.15 \times 10^{-3} \\
5.19 \times 10^{-3} \\
5.10 \times 10^{-3} \\
6.40 \times 10^{-3} \\
8.28 \times 10^{-3} \\
11.30 \times 10^{-3} \\
13.10 \times 10^{-3} \\
18.00 \times 10^{-3} \\
25.80 \times 10^{-3} \\
40.50 \times 10^{-3}\end{aligned}$ & $\begin{array}{r}6 \\
10 \\
11 \\
17 \\
24 \\
25 \\
24 \\
23 \\
25 \\
21 \\
17 \\
13 \\
10 \\
5 \\
11 \\
13 \\
21 \\
26 \\
33 \\
35 \\
39 \\
41 \\
35 \\
34 \\
36 \\
39 \\
36 \\
33 \\
31\end{array}$ & $\begin{array}{r}\% \\
2.8 \\
3.1 \\
2.5 \\
2.1 \\
2.1 \\
2.2 \\
1.9 \\
2.2 \\
2.3 \\
3.2 \\
5.2 \\
4.0 \\
5.9 \\
8.0 \\
5.4 \\
4.5 \\
3.4 \\
2.5 \\
2.2 \\
2.1 \\
2.0 \\
2.1 \\
1.9 \\
1.8 \\
1.8 \\
1.6 \\
1.7 \\
1.9 \\
1.9\end{array}$ \\
\hline
\end{tabular}

$\alpha=\sigma_{\bar{\tau}}^{-} / \bar{\tau} \times 100$.

The table shows that these measurements of refraction are consistent with the form of the expected variation of refraction. That is, at low angles, refraction is large (on the order of $0.5 \mathrm{deg}$ ) and it becomes very small at high-altitude angles, being minimum at meridian passage. Plots of the average refraction and standard error of estimate of the average versus observed altitude angle for a.m. and p.m. are shown in figures 1 and 2 .

The well-behaved smooth variation of $\bar{\tau}$ with altitude angle suggests that the observations were indeed measurements of refraction and that removal of extraneous effects has been rather successfully accomplished. Furthermore, it suggests that the radio sextant has been able to perform these measurements with high precision.

The standard error of estimate of refraction remains just slightly less than $0.001 \mathrm{deg}$ for all angles greater than $20 \mathrm{deg}$. Below $20 \mathrm{deg}$ the standard error of estimate of the average increases with de- 
creasing altitude angle. This undoubtedly results primarily from the increasing variability of refraction at low angles, and to a secondary extent, from the decreasing number of observations at very low angles.

Table 1 and figures 3 and 4 show the standard deviation of the measured values of refraction for each altitude angle. The gross variation is similar to refraction in that the standard deviation is small at high-altitude angles and increases as altitude angle decreases.

There are four factors which contribute to this increase of variability at low angles. The first involves the tracking system behavior with variation of loop gain. Tracking-servo random errors increase as the difference between the power received from the sun and the surrounding sky decreases. At low-altitude angles, the sun's radiation must pass through a greater distance of attenuating atmosphere than at high angles. This results in an appreciable reduction of power received from the sun at low angles. In addition, energy emitted from the atmosphere itself increases as the transmission path length increases. Although this second effect is very small, it may contribute to the less precise ability to distinguish between the sun and the surrounding sky at very low angles. The increasing path length, in effect, increases the tracking-servo random errors at low angles.

The second factor is the rapidly changing meteorological conditions at the sunrise and sunset periods. At sunrise, insolation produces heating of the earth's surface and causes parcels of surface air to be heated and then to rise by convection. In addition to causing rapid variation in the surface refractivity, this heating from below also produces a convectively mixed lower atmosphere. As insolation decreases in the afternoon, convective mixing ceases, the surface temperature begins to decrease, and the atmosphere begins to settle and stratify into more uniformly varying layers. Near sunset, surface refractivity is again changing appreciably, and the atmosphere is less homogeneous.

The third factor contributing to increased variability at low angles is the fact that the refractivity profile becomes more important at low angles. Thus, the state of the atmosphere above the surface contributes to the total refraction. Furthermore, as the altitude angle decreases, the ray path extends over greater distances from the observing station.

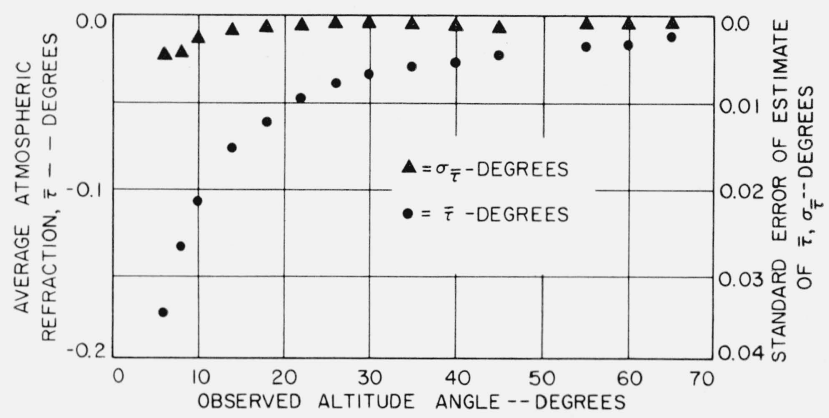

Figure 1. Average atmospheric refraction and standard error of estimate obtained during a.m.

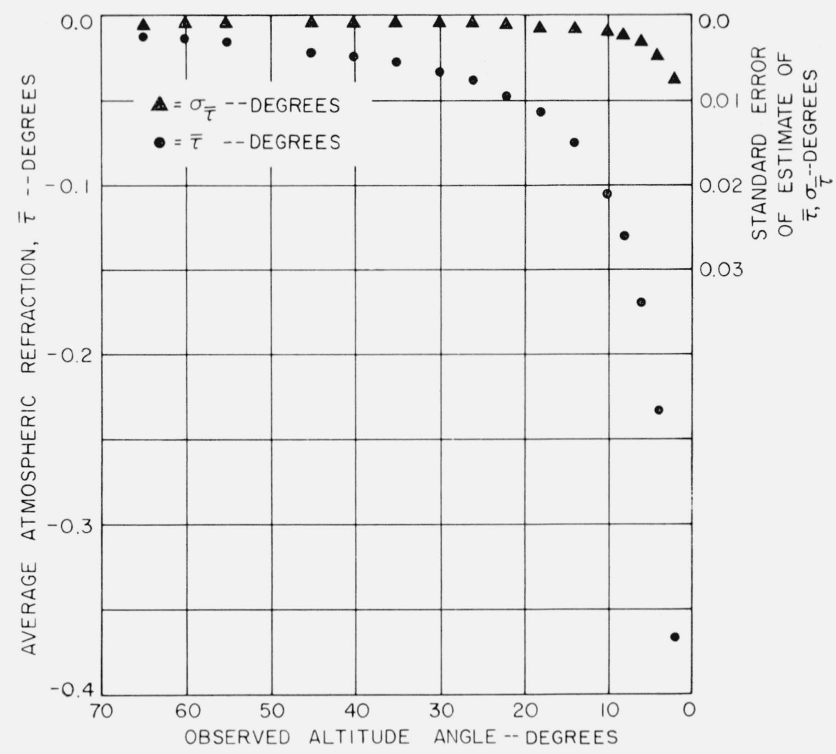

Figure 2. Average atmospheric refraction and standard error of estimate obtained during p.m.

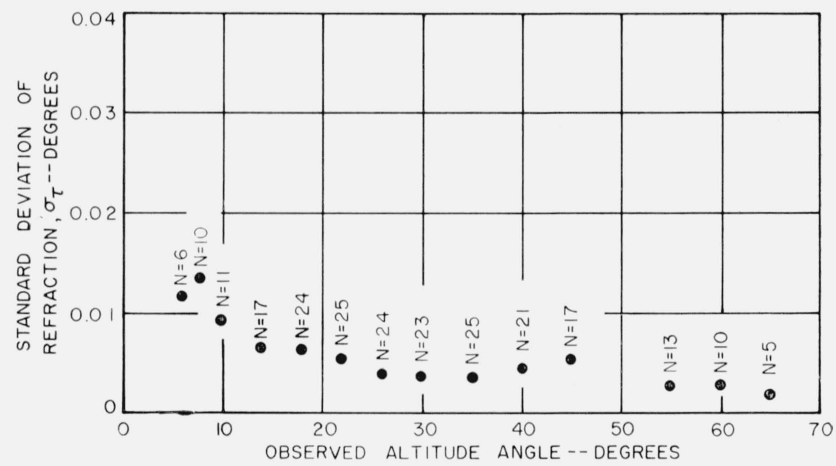

FIGURE 3. Standard deviation of refraction for a.m. observations.

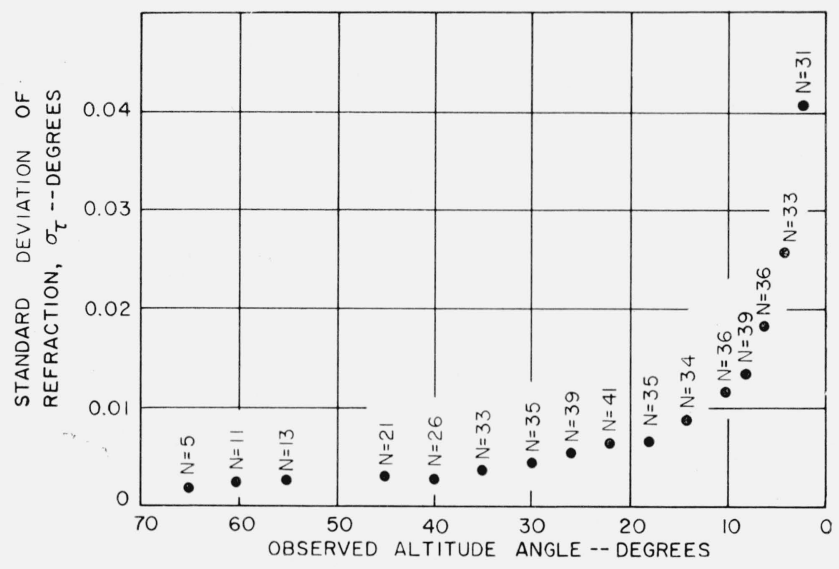

FIGURE 4. Standard deviation of refraction for p.m. observations. 
Thus, nonlocal variations in meteorology have an increasing effect on total refraction.

The final contribution to variability of total refraction results from the inability to obtain measurements of refraction at exact integral values of observed altitude angle. Since the various values of observed angle were symmetrically distributed about the desired integral value, no systematic bias was introduced, but some of the variability in $\sigma_{\tau}$ results from this variability in $h_{0}$. The fact that $h_{0}$ was truncated at $\pm 0.09 \mathrm{deg}$ from the desired value permits some estimate of the rms variability of the error in $h_{0}, \sigma_{\Delta h_{0}}$, to be calculated in the usual manner by averaging the sum of squares of error. Thus,

$$
\begin{aligned}
\sigma_{\Delta h_{0}}^{2} & =\frac{1}{\Delta} \int_{h_{0}^{\prime}}^{h_{0}^{\prime}+\Delta}\left(h_{0}^{\prime}-h_{0}\right)^{2} d h_{0} \\
& =\frac{\Delta^{2}}{3} \\
\sigma_{\Delta h_{0}} & =\frac{\Delta}{\sqrt{3}}=\frac{0.09}{1.73}=0.052 \mathrm{deg},
\end{aligned}
$$

where $h_{0}^{\prime}$ is the desired integral value of observed altitude and $\Delta$ is the one-way maximum error $(0.09$ deg). Using the slope of the $\tau$ versus $h_{0}$ curve, the variability in $h_{0}$ can be converted into variability in $\tau$. Thus,

$$
\sigma_{\tau}^{\prime}=\left|\frac{d \tau}{d h_{0}}\right| \sigma_{\Delta h_{0}} \operatorname{deg} .
$$

The magnitude of the rate of change, $\left|d \tau / d h_{0}\right|$, can be found from the graph of refraction, which gives the maximum rate magnitude as $0.066 \mathrm{deg}$ per degree at $2 \mathrm{deg}$ altitude angle. Thus, the maximum estimated variability in refraction due to variability in selecting $h_{0}$ is $\sigma_{\tau}^{\prime}=0.0034 \mathrm{deg}$. This represents 8.4 percent of the total variability in $\tau$ at $2 \mathrm{deg}$ altitude.

In figure 5 are plotted the various values of the percent error of estimate of total refraction. The graph shows minimum percent error at the low altitude angles with a general increase to the maximum of 8 percent at $65 \mathrm{deg}$. This behavior arises from the fact that the standard error of estimate reaches a minimum value at high angles, but the total refraction continues to decrease monotonically.

The statistical tests described earlier, indicated that a.m. refraction was greater than p.m. refraction. Comparison of the two values for each altitude angle shown in table 1 verifies that this is consistently true. The differences are plotted as a function of altitude angle in figure 6 . The dashed line indicates the average difference of $0.0025 \mathrm{deg}$. While this investigation did not attempt to determine why this is so, it is evidently true, and the following hypothesis is offered without verification. As a result of asymmetry in the diurnal temperature cycle, morning hours are generally cooler than afternoon. Since refractivity is inversely proportional to temperature, the surface refractivity would be expected to be somewhat greater during the morning hours. Figure 7 shows the average surface refractivity over a month for each hour of the day. The diurnal variation is clearly evident and shows that, on the average, a.m. surface refractivity in fact is greater than p.m. The greater surface refractivity and the more homogeneous mixing would tend to produce more refractive bending in the first few kilometers above the surface during the morning hours.

Comparisons of measured radio refraction with standard optical refraction from the Nautical Almanac, $8.7-\mathrm{mm}$ measured refraction, and that calculated by ray tracing from an assumed model atmosphere are shown in figure 8. This figure shows that the refraction measured by the radio sextant at $1.85 \mathrm{~cm}$ is consistently greater than any of the other determinations of atmospheric refraction. This is probably not a deficiency in the measurements described in this paper, but rather, is the

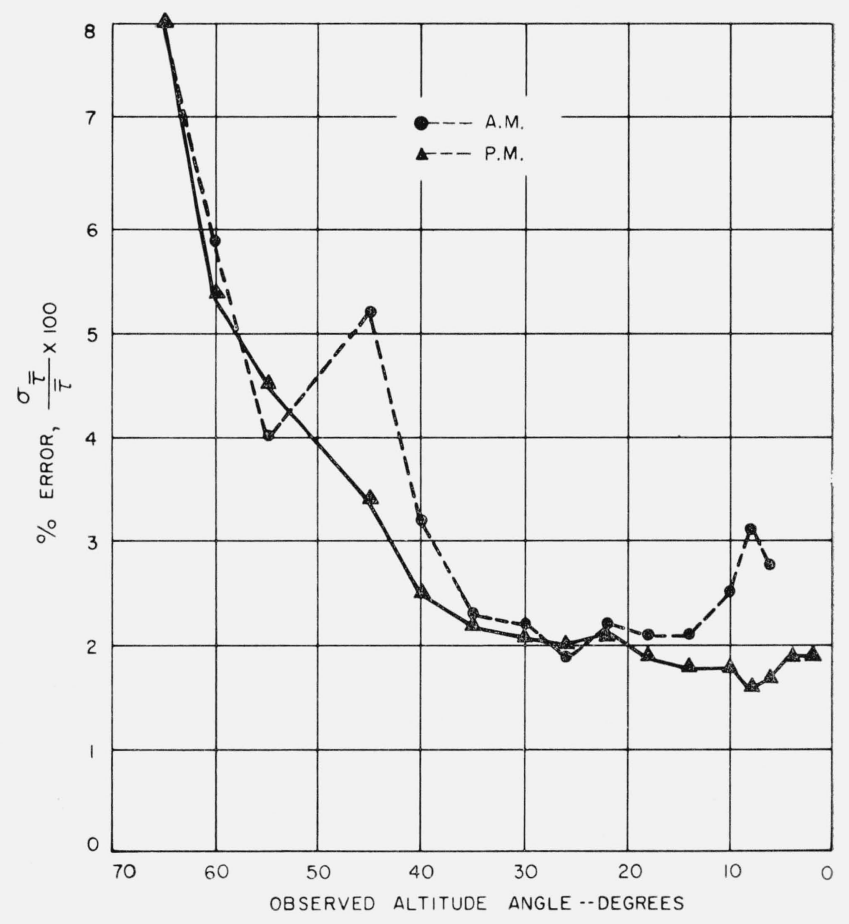

Figure 5. Percent error of estimate of total refraction.

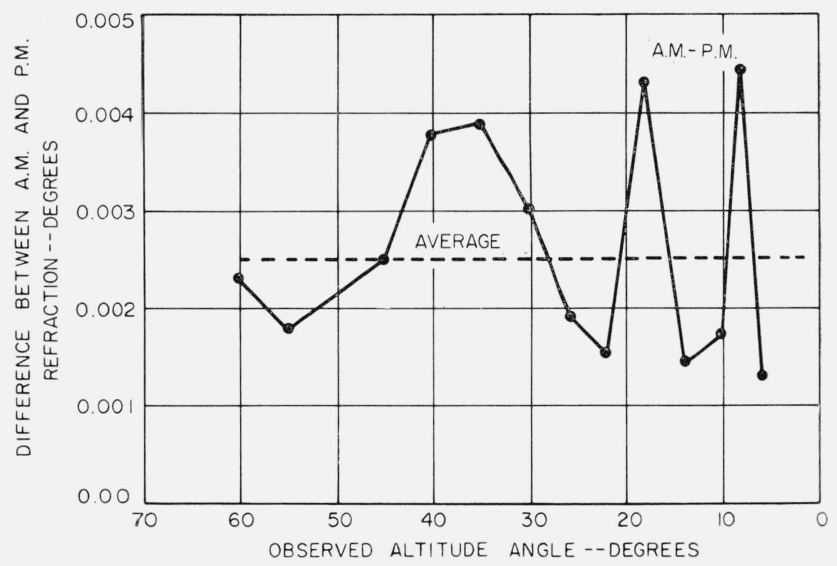

Figure 6. Difference between a.m. and p.m. refraction. 
result to be expected in view of the facts that the average surface refractivity during the sampling period was relatively high, and that $1.85-\mathrm{cm}$ radio refractivity is higher than that at optical wavelengths. The latter results from the decreased dielectric polarization of the water vapor molecule.

The difference between radio and standard optical refraction is seen to be appreciable even at highaltitude angles. Since the optical refraction was determined for standard conditions of temperature and pressure, it is believed that some reduction of this difference would be obtained if optical refractivity were determined for the nonstandard conditions obtained during observation. Optical refractivity does not include the contribution of water vapor, so a fundamental difference will exist no matter how well the optical refraction may be determined. Recent publications of the Nautical Almanac do have tables which provide further correction to the standard optical refraction for nonstandard conditions.

Calculated refraction gives the closest comparison with the measured results. The calculations were performed for a nonreal atmosphere with a surface refractivity of $318 \mathrm{~N}$-units, which is about 4.3 percent less than the average measured surface refractivity during the experimental period. In other words, if the assumed model profile had a surface refractivity of $332 N$-units, the measured values of total refraction would then compare more favorably, especially at the higher altitude angles.

The second general feature of figure 8 is increasing discrepancy in all cases at angles below approximately 20 degrees. This behavior is primarily a consequence of the difference in surface refractivity between the measured data and the optical and assumed models and, to a lesser extent, of the dependence, at low angles, of total refraction on the refractivity profile. That is, the total discrepancy at low angles is not accounted for by the difference in surface refractivity alone. A small portion of the low-angle discrepancy is attributed to difference in shape of the refractivity profiles for the measured data and the assumed models.

Peculiar behavior of the $8.7-\mathrm{mm}$ difference plot is believed due primarily to the assumption by Marner and Ringoen [1956] that change in refraction is negligible above about $30 \mathrm{deg}$. The inflection around $10 \mathrm{deg}$ probably results from systematically readjusting the tracking servo bias at $10 \mathrm{deg}$ altitude angle.

Theoretical considerations indicate that total refraction is a linear function of surface refractivity for reasonably high altitude angles [Bean and Cahoon, 1957]. To test the hypothesis that this relation holds for low-altitude angles, the typical results shown in figure 9 were obtained. At 8 deg altitude, the linear correlation coefficient was 0.87 , which indicates a high degree of confidence in the linear hypothesis. Furthermore, the standard error of prediction is reduced from 0.014 to 0.007 by use of the linear predictor. There is little apparent curvature in the distribution of these sample points, which gives further support to the hypothesis.

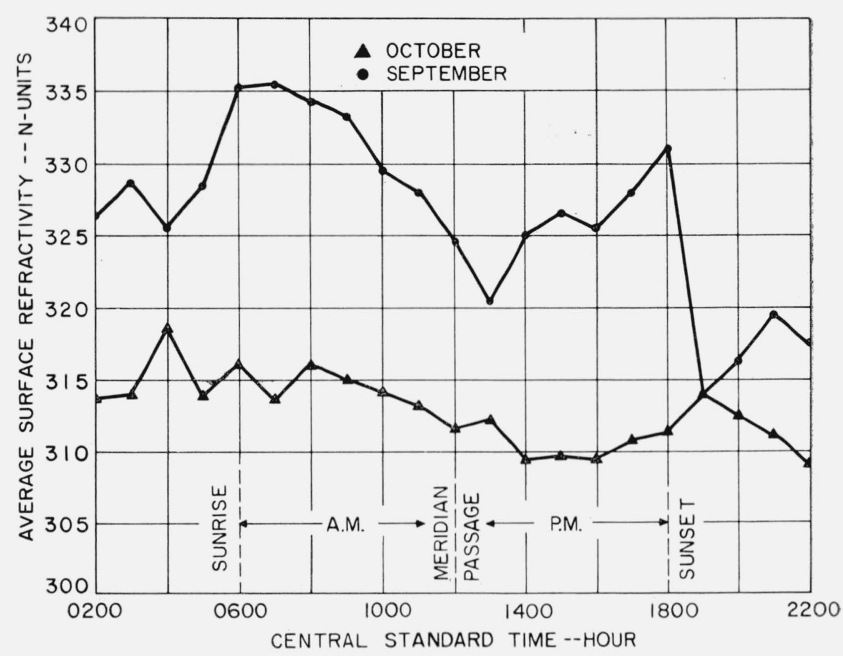

Figure 7. Average surface refractivity.

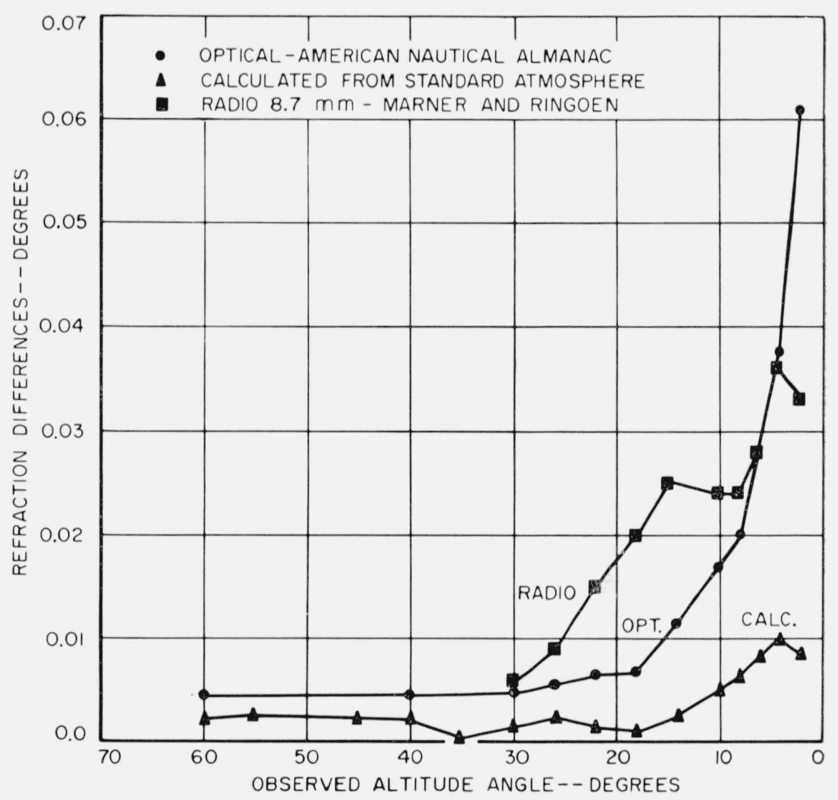

Figure 8. Comparison of refraction differences $(1.85 \mathrm{~cm}$ measured value minus specified value).

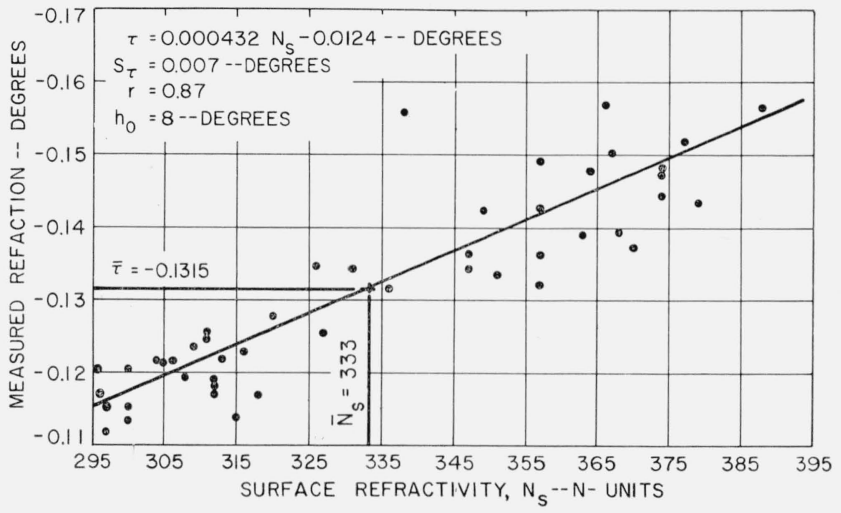

FiguRE 9. Correlation of total refraction with surface refractivity. 


\section{Conclusions}

A precise radiometric method of determining the average total atmospheric refraction at a $1.85-\mathrm{cm}$ wavelength has been described. The maximum standard error of estimate of refraction was 0.0073 deg at 2 deg altitude angle, and the minimum was $0.0006 \mathrm{deg}$ at $35 \mathrm{deg}$ altitude angle. Expressed in terms of percentage of the mean, the error of estimate of the mean ranged from 8.0 percent to 1.6 percent. Less than 8.4 percent of the total errors of measurement were due to the inability to make the observations precisely at the desired altitude angle. Errors due to this cause were maximum at the lowest altitude angles and decreased rapidly with increasing altitude angle.

The specinic determinations of refraction obtained during this investigation are valid only for August through December 1959 at Cedar Rapids, Iowa. Any extrapolation for other time or location must be used with care. If the average surface refractivity for some other location and time is known, these results, could be used by modifying them in the ratio of that average value to $332 N$-units. Such a procedure should give a valid first approximation to the average radio refraction for a new time and location.

Probably the most significant conclusion which can be drawn from this investigation is the support which it gives to the various theoretical considerations which have been advanced. Notably among them is the analytical solution given by Smart [1956] and the linear relationship between surface refractivity and total atmospheric refraction suggested by Bean and Cahoon [1957]. The success of these measurements also contributes considerable weight to the efficacy of the Smith and Weintraub [1953] equation for radio refractivity.

This investigation has brought into somewhat sharper focus two particular results. They are the difference between a.m. and p.m. refraction at the same observed altitude angle, and the dispersion of radio refractivity. Both of these phenomena have theoretical bases and the present results suggest that further investigation would be warranted.

The results reported here are strictly long-term average values and must not be interpreted as having any particular relation to the "instantaneous" refraction under other observing conditions. The results do, however, indicate the gross variational pattern and supply information on the range of variation to be expected throughout the fall months in the midwestern United States.

\section{References}

Aarons, J., W. R. Barron, and J. P. Castelli (Jan. 1958), Radio astronomy measurements at VHF and microwaves, Proc. IRE 46, 325-333.

Anderson, W. L., N. J. Beyers, and R. J. Rainey (Sept. 1960), Comparison of experimental with computed tropospheric refraction, IRE Trans. Antennas Propagation, AP-8, 456461.

Bean, B. R., and B. A. Cahoon (July 1957), The use of surface weather observation to predict the total atmospheric bending of radio rays at small elevation angles, Proc. IRE 45, No. 11, 1545-1546.

Marner, Gene R., and Richard M. Ringoen (March 20, 1956), Atmospheric refraction of $8.7 \mathrm{Mm}$ radiation. National IRE Convention.

Marner, Gene R., and Clyde Stewart (June 1955), Final report development of $8.7 \mathrm{Mm}$ wavelength radio sextant and associated studies, Collins Engineering Report No. CER 409, Cedar Rapids, Iowa, Collins Radio Co.

Ringoen, R. M., Wm. H. Bellville, and R. J. LeMont (June 1952), Final Report on the microwave radiometry project, Collins Research Report No. CRR 122, Cedar Rapids, Iowa, Collins Radio Co.

Schulkin, M. (May 1962), Average radio-ray refraction in the lower atmosphere, Proc. IRE 40, 554-561.

Smart, W. (1956), Spherical astronomy, Colchester, England (Cambridge Press).

Smith, E. K., Jr., and Stanley Weintraub (Aug. 1953), The constants in the equation for atmospheric refraction index at radio frequencies, Proc. IRE 41, 1035-1037.

(Paper 67D2-251 